\title{
On the Relation between Composite Right-/Left-Handed Transmission Lines and Chebyshev Filters
}

\author{
Changjun Liu ${ }^{1}$ and Wolfgang Menzel $^{2}$ \\ ${ }^{1}$ The School of Electronics and Information Engineering, Sichuan University, Chengdu 610064, China \\ ${ }^{2}$ The Institute of Microwave Techniques, University of Ulm, $89069 \mathrm{Ulm}$, Germany \\ Correspondence should be addressed to Changjun Liu, cjliu@ieee.org
}

Received 16 July 2009; Accepted 23 October 2009

Recommended by Kamya Yekeh Yazdandoost

Composite right-/left-handed (CRLH) transmission lines have gained great interest in the microwave community. In practical applications, such CRLH sections realized by series and shunt resonators have a finite length. Starting from the observation that a high-order Chebyshev filter also exhibits a periodic central section of very similar structure, the relations between finite length CRHL transmission lines and Chebyshev filters are discussed in this paper. It is shown that a finite length CRLH transmission line in the balanced case is equivalent to the central part of a low-ripple high-order Chebyshev band-pass filter, and a dual-CRLH transmission line in the balanced case is equivalent to a low-ripple high-order Chebyshev band-stop filter. The nonperiodic end sections of a Chebyshev filter can be regarded as matching sections, thus leading to an even better amplitude and phase response. It is also shown that, equally to a CRHL transmission line, a Chebyshev filter exhibits negative phase velocity in part of its passband. As a consequence, an improved behavior of finite length CRLH transmission lines may be achieved adding matching sections based on filter theory; this is demonstrated by a simulation example.

Copyright (C) 2009 C. Liu and W. Menzel. This is an open access article distributed under the Creative Commons Attribution License, which permits unrestricted use, distribution, and reproduction in any medium, provided the original work is properly cited.

\section{Introduction}

In the past years, metamaterials [1] have found wide interest in the microwave community. A homogeneous negative refractive index, left-handed, or metamaterial transmission line, however, does not exist in nature and typically is approached by an artificial structure which is usually constructed from a series of discontinuous sections and operates in a restricted frequency range $[2,3]$. When the dimension of such sections is much smaller than the wavelength at the operating frequencies, such transmission lines are considered as homogeneous media. A typical realization is found in a quasilumped transmission line with elementary cells consisting of series capacitors and shunt inductors [4]. As the normal shunt capacitance and series inductance cannot be avoided in practice, the concept of a composite right/left-handed (CRLH) transmission line was developed, and a number of applications have been demonstrated [49], for example, for frequency-scanned antennas, directional couplers, power dividers, zeroth-order resonators, and so forth, and further applications of negative refractive index transmission lines for constructing advanced microwave components $[10,11]$ are envisaged.

Figure 1(a) shows the equivalent circuit of a (finite length) CRLH transmission line (connected to a source generator on the left side and a load at the right side). Looking at this equivalent circuit, this CRLH transmission line resembles a band-pass filter of typically high order. In the following, we limit our discussions to such one-dimensional CRLH transmission lines.

In a dual-CRLH transmission line, the series-branch and the shunt-branch resonators of a CRLH transmission line are interchanged resulting in a different type of negative refractive index transmission line [12]. Figure 1(b) shows the circuit model of such a dual-CRLH transmission line, which, in this case, looks like a high-order band-stop filter.

A conventional filter, on the other hand, is generally not a uniform structure. However, looking at the filter coefficients $g_{i}$ of a Chebyshev filter, such a filter with high order exhibits a highly periodic central section. From a different point of 


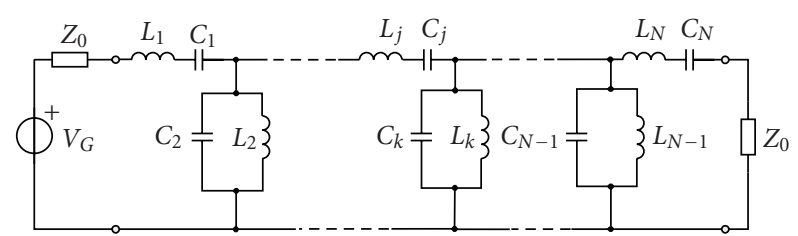

(a)

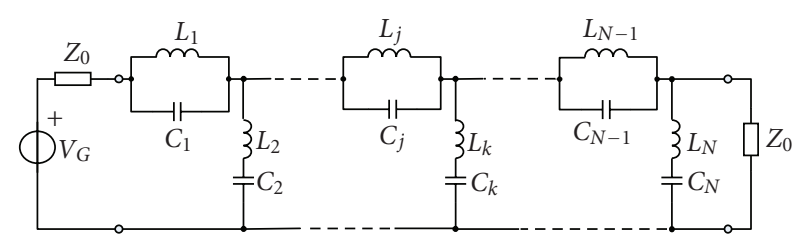

(b)

Figure 1: Equivalent circuit models of two finite length negative refractive index transmission lines. (a) CRLH transmission line. (b) Dual-CRLH transmission line.

view, it also can be considered as a periodic structure with impedance matching sections at both ends (see Figure 3). In [13] we have briefly shown that the CRLH transmission line may be equivalent to a part of the Chebyshev band-pass filter, which was done partly in an intuitive way.

The starting points of our considerations were on the one hand, the periodic nature of the central part of Chebyshev filters, and on the other hand, practical problems of implementing finite length CRLH transmission lines into a (typically) $50 \mathrm{Ohm}$ environment. Therefore, we wanted to find the relations between CRLH transmission lines and Chebyshev filters to design better CRLH transmission line sections with the help of classical filter theory.

Typically, CRLH transmission lines are considered as tools for dispersion engineering-this is in some way equivalent to frequency dependent phase control. Amplitude plays a minor role, although the practical implementation of a finite length section of a CRLH transmission line (and only this is feasible in reality) leads to problems with impedance match. On the other hand, filters are normally considered as components which control the amplitude performance of a signal while maintaining a good impedance match. Looking at many system applications, however, group delay (and therefore phase behavior as a function of frequency) plays an important role as well-there are even special filter design procedures strongly concentrating on group delay/phase behavior (e.g., Bessel or linear phase filters). This indicates closer relations between the two concepts than usually perceived.

In this paper, we deepen and extend the discussion in [13] and compare the design of CRLH transmission lines with the design of high-order Chebyshev filters in more detail. It is proven that a finite length CRLH transmission line can be considered as the central part of a Chebyshev filter. Thus, if we design a CRLH transmission line based on the classical filter theory, a better performance of a finite length transmission line section can be achieved. To this end, we firstly summarize the characteristic formulas of a CRLH transmission line. Secondly, we recall the design formulas for a Chebyshev band-pass filter with a similar characteristic and prove that the central section of the Chebyshev band-pass filter is identical to a finite section of a CRLH transmission line. By attaching the ending sections of the filter, a finite length CRLH transmission line with better performance is achieved. A similar result can be obtained for dual-CRLH transmission lines. Thirdly, we compare the group delay and phase distribution between a CRLH transmission line and a Chebyshev filter. Backward wave propagation inside a Chebyshev filter is indicated. Finally, we present an example on how to improve the design of CRLH transmissions lines in a simple way.

\section{CRLH Transmission Line in the Balanced Case}

The equivalent circuit of a CRLH transmission line is a cascaded network as shown in Figure 1(a) which consists of series $L C$ resonators alternating with shunt $L C$ resonators. It is a periodic structure, and the $L C$ components are

$$
\begin{aligned}
& L_{j}=L_{R}, \quad C_{j}=C_{R}, \quad \text { for series branch resonators, } \\
& L_{k}=L_{L}, \quad C_{k}=C_{L}, \quad \text { for shunt branch resonators, }
\end{aligned}
$$

where the subscripts $L$ and $R$ denote the "left-handed" and the "right-handed" cases, respectively. The resonant frequencies of the left-/right-handed $L C$ circuits are $\omega_{L}=$ $1 / \sqrt{L_{L} C_{L}}$ and $\omega_{R}=1 / \sqrt{L_{R} C_{R}}$. The resonant frequencies of the series-branch resonators and shunt-branch resonators are $\omega_{\mathrm{se}}=1 / \sqrt{L_{R} C_{L}}$ and $\omega_{\mathrm{sh}}=1 / \sqrt{L_{L} C_{R}}$. We define one series $L C$ resonator $L_{j} C_{j}$ together with one adjacent shunt $L C$ resonator $L_{k} C_{k}$ as a unit cell to analyze the periodic structure. The characteristic impedance $Z_{C}$ of the CRLH transmission line obtained from the image impedance analysis [14] is

$$
Z_{C}=\sqrt{Z_{L} Z_{R} \frac{\varepsilon_{\mathrm{se}}}{\varepsilon_{\mathrm{sh}}}\left(1-\frac{\varepsilon_{\mathrm{se}} \varepsilon_{\mathrm{sh}}}{4} \frac{\omega_{L}}{\omega_{R}}\right)},
$$

where $\varepsilon_{\mathrm{se}}=\omega / \omega_{\mathrm{se}}-\omega_{\mathrm{se}} / \omega, \varepsilon_{\mathrm{sh}}=\omega / \omega_{\mathrm{sh}}-\omega_{\mathrm{sh}} / \omega, Z_{L}=$ $\sqrt{L_{L} / C_{L}}$, and $Z_{R}=\sqrt{L_{R} / C_{R}}$. If $\varepsilon_{\mathrm{Se}}=\varepsilon_{\mathrm{Sh}}$ holds (equivalent to $\left.\omega_{\mathrm{se}}=\omega_{\mathrm{sh}}\right)$, the band gap between the left-/righthanded regions vanishes. This case is often referred to the balanced case of CRLH transmission lines. A comparison of the characteristic impedance of a CRLH transmission line between the balanced and unbalanced case is shown in Figure 2. Even in the balanced case, a perfect impedance match can be only achieved at the transition frequency $\omega_{0}$.

The balanced condition of a CRLH transmission line is

$$
\omega_{\mathrm{Se}}=\omega_{\mathrm{Sh}}=\omega_{0} \quad \text { or } \quad L_{L} C_{R}=L_{R} C_{L},
$$

where $\omega_{0}$ is the transition angular frequency between the left/right-handed regions. The same results can be found in [5, $6]$.

In the balanced case, the characteristic impedance of the CRLH transmission line is

$$
Z_{C}=Z_{0} \sqrt{1-\frac{\varepsilon^{2}}{4} \frac{\omega_{L}}{\omega_{R}}},
$$




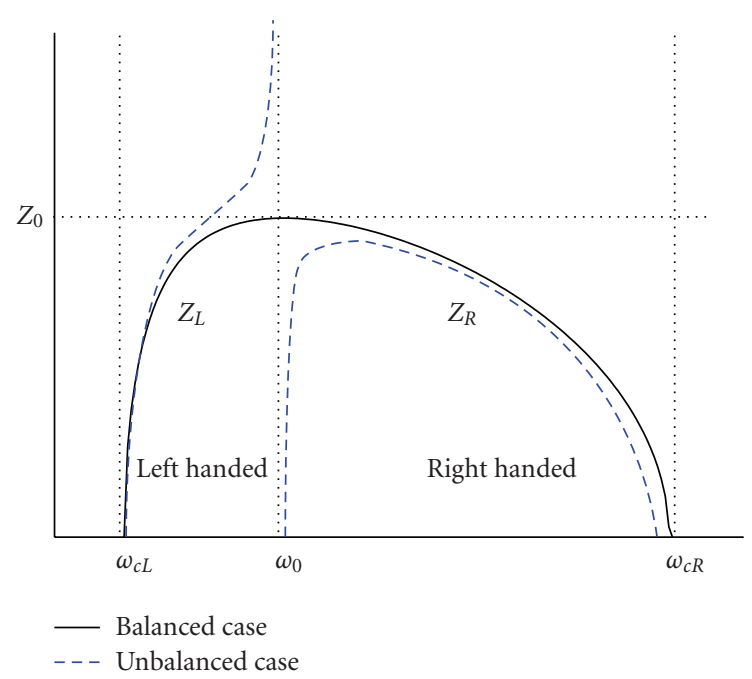

FIgURe 2: Characteristic impedance of a CRLH transmission line in the balanced/unbalanced case.

where $\varepsilon=\omega / \omega_{0}-\omega_{0} / \omega$ and $Z_{0}=Z_{L}=Z_{R}$. Assuming an infinitely long CRLH transmission line in the balanced case, the passband of the CRLH transmission line is corresponding to $Z_{C}>0$. Thus, the lower and upper cut-off frequencies are determined by the condition $Z_{C}=0$. We obtain the corresponding equation on $\omega$ from (4) as

$$
\frac{1}{4}\left(\frac{\omega}{\omega_{0}}-\frac{\omega_{0}}{\omega}\right)^{2} \frac{\omega_{L}}{\omega_{R}}=1 .
$$

Then, we obtain the lower and higher cut-off frequencies by solving (5) as

$$
\begin{aligned}
& \omega_{c L}=\omega_{R}\left(\sqrt{1+\frac{\omega_{L}}{\omega_{R}}}-1\right), \\
& \omega_{c R}=\omega_{R}\left(\sqrt{1+\frac{\omega_{L}}{\omega_{R}}}+1\right) .
\end{aligned}
$$

From (3) and (5), the relation between the transition frequency and cut-off frequencies is

$$
\omega_{0}^{2}=\omega_{c L} \omega_{c R}=\omega_{\mathrm{sh}} \omega_{\mathrm{se}}=\frac{1}{\sqrt{L_{L} L_{R} C_{L} C_{R}}} .
$$

There are four independent parameters, namely, $L_{L}, C_{L}$, $L_{R}$, and $C_{R}$, in a CRLH transmission line design. With the balanced condition, only three independent parameters can be chosen freely. Once two cut-off frequencies and the characteristic impedance $Z_{C}$ (matching to $Z_{0}$ ) are fixed, a unique CRLH transmission line configuration is determined by (5) and (6) by subtracting the two equations in (5) and introducing $Z_{0}$ and $\omega_{0}$ :

$$
\begin{aligned}
& \omega_{0} L_{R}=\frac{1}{\omega_{0} C_{L}}=\frac{\omega_{0}}{\omega_{c R}-\omega_{c L}} 2 Z_{0}, \\
& \omega_{0} C_{R}=\frac{1}{\omega_{0} L_{L}}=\frac{\omega_{0}}{\omega_{c R}-\omega_{c L}} \frac{2}{Z_{0}} .
\end{aligned}
$$

\section{Chebyshev Band-Pass Filter}

An $N$ th-order band-pass filter in principle has the same $L C$ equivalent circuit as the CRLH transmission line in Figure 1(a). The band-pass filter design is usually achieved from the lowpass to band-pass transformation, in which a prototype lowpass filter is applied. The mapping formulas can be found in [14] with

$\omega_{0} L_{j}=\frac{1}{\omega_{0} C_{j}}=\frac{\omega_{0}}{\omega_{2}-\omega_{1}} g_{j} Z_{0}$, for series branch resonators,

$\omega_{0} C_{k}=\frac{1}{\omega_{0} L_{k}}=\frac{\omega_{0}}{\omega_{2}-\omega_{1}} \frac{g_{k}}{Z_{0}}$, for shunt branch resonators,

where $g_{i}$ is the $i$ th element value of the prototype lowpass filter, $\omega_{1}$ and $\omega_{2}$ are the lower and upper cut-off frequencies, respectively, and $\omega_{0}=\sqrt{\omega_{1} \omega_{2}}$ is the center frequency. $Z_{0}$ is the system impedance. Once $g_{i}, \omega_{1}, \omega_{2}$, and $Z_{0}$ are fixed, a band-pass filter is uniquely determined by (8).

From (8) we can obtain

$$
L_{j} C_{j}=L_{k} C_{k}=\frac{1}{\omega_{0}^{2}}
$$

which is equivalent to (3). In a band-pass filter design, the balanced case of a CRLH transmission line always holds.

Since the mapping from a prototype lowpass to a bandpass filter is a generic formula, it can be applied to any type of prototype lowpass filter. Thus, the balanced case of a CRLH transmission line is automatically realized in bandpass filters from any kind of prototype lowpass filters with series- $L C$ and shunt- $L C$ resonators. Butterworth, Gaussian, or Chebyshev (with any passband ripples) band-pass filters constructed from (8) satisfy the balanced condition of a CRLH transmission line.

In most prototype lowpass filters, the element values $g_{i}$ usually vary in a certain range and lead to a nonperiodic structure. The central section of a high-order Chebyshev filter, however, has a periodic structure. Figure 3 shows an example of a 41st-order Chebyshev prototype lowpass filter with several values of passband ripple. For elements not close to either end, element values are practically periodic. It should be noticed that always two adjacent filter elements form one equivalent transmission line cell. Thus, the central part of a Chebyshev is a quasiperiodic structure, independently of the passband ripple.

3.1. Low Passband Ripple. The element values of a 41st-order Chebyshev prototype lowpass filters with several different values of passband ripple are shown in Figure 3. The lower the passband ripple is, the lower is the variation of the element values. In the limiting case, the values of the central elements are close to $g_{i}=2$. When the passband ripple in a Chebyshev lowpass filter is low and its order is high, it can be proven theoretically or shown numerically that the filter element values approach $g_{i}=2$ for all elements that are not close to either filter end. 


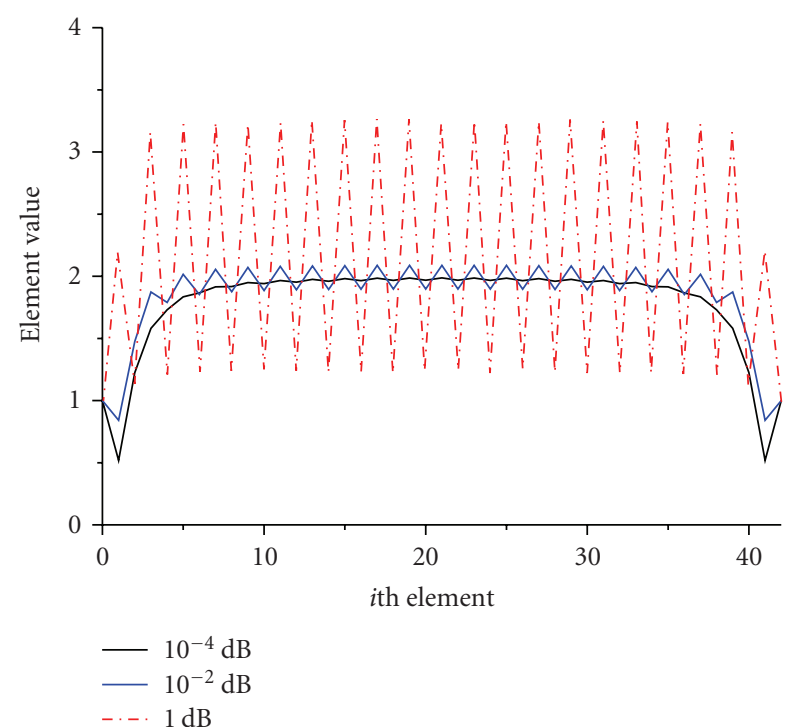

FIGURE 3: Element values of 41st-order prototype Chebyshev lowpass filters with different passband ripples.

When the element value is $g_{j}=g_{k}=2$, (7) and (8) become equivalent to each other. Thus, if a Chebyshev band-pass filter and a CRLH transmission line have the same cut-off frequencies (i.e., $\omega_{1}=\omega_{c L}, \omega_{2}=\omega_{c R}$ ) and system impedance $Z_{0}$, the same $L C$ equivalent circuit is determined by either (7) or (8). Once $\omega_{1}, \omega_{2}$, and $Z_{0}$ are fixed, the corresponding CRLH transmission line in the balanced case and the central part of the corresponding high-order Chebyshev band-pass filter with low passband ripple have identical $L C$ configurations.

3.2. High Passband Ripple. In a high-order Chebyshev prototype lowpass filter with larger passband ripple, element values oscillate around $g=2$ as shown in Figure 3. For those central elements, element values of series-branch $L C$ resonators and shunt-branch $L C$ resonators keep constant with $g_{\text {se }}$ and $g_{\text {sh }}$, alternatively, and $g_{\text {se }} \cdot g_{\text {sh }} \approx 4$ always holds. Thus, a high-ripple high-order Chebyshev band-pass filter has a periodic configuration as well. It is a modified balanced CRLH transmission line which will be discussed in Section 4 .

Once two cut-off frequencies and the system impedance are given, a uniform CRLH transmission line in the balanced case and a high-order Chebyshev band-pass filter with low passband ripple can be uniquely implemented with $L C$ circuits, respectively. In other words, a finite length uniform CRLH transmission line in the balanced case is equivalent to the central part of a high-order low-ripple Chebyshev band-pass filter with the same cut-off frequencies and system impedance.

\section{Impedance Match and Passband Ripple}

We first consider a CRLH transmission line in the balanced case. The characteristic impedance is dependent on frequency as in (4), and the calculated results are shown in Figure 2. A match between the CRLH transmission line and the system impedance $Z_{0}$ can be obtained only around the transition frequency $\omega_{0}$. This indicates that impedance matching circuits should be applied in broadband designs and applications.

When the passband ripple of a Chebyshev prototype lowpass filter is high, the element values of the central part are alternatively $g_{\mathrm{se}}=2 \alpha^{2}$ and $g_{\mathrm{sh}}=2 / \alpha^{2}$ for series and shunt branches, where $\alpha$ is a coefficient dependent on the passband ripple. If $Z_{0}$ in (7) is replaced by $\alpha Z_{0}$, it is still a well-designed CRLH transmission line. Therefore, the passband ripple of a Chebyshev band-pass filter corresponds to an impedance mismatch between the applied and designed system impedance of the CRLH transmission line. The greater the difference is, the higher is the passband ripple. Based on the analysis of element values of a high-order Chebyshev prototype lowpass filter, the relation between the passband ripple and the impedance mismatch is

$$
\alpha=\left(\frac{g_{\mathrm{se}}}{g_{\mathrm{sh}}}\right)^{s / 2}=\operatorname{coth}^{s}\left(\frac{1}{2} \sinh ^{-1}\left(10^{L_{A r} / 10}-1\right)^{-1 / 2}\right),
$$

where $L_{A r}$ is the passband ripple in $\mathrm{dB}$, and the sign function is

$$
s= \begin{cases}+1, & Z_{0}^{\text {Designed }}>Z_{0}^{\text {Applied }} \\ -1, & Z_{0}^{\text {Designed }}<Z_{0}^{\text {Applied }} .\end{cases}
$$

Once $\alpha$ is given, the passband ripple can be obtained from (10). For example, if the external system impedance is $50 \Omega$ and the characteristic impedance of a CRLH transmission line is $70 \Omega$, the corresponding passband ripple of the bandpass filter is $L_{A r}=0.4827 \mathrm{~dB} \approx 0.5 \mathrm{~dB}$.

Therefore, the central part of a high-ripple high-order Chebyshev band-pass filter is equivalent to a CRLH transmission line with mismatched impedance to the source and load.

When the external and the design impedances of a CRLH transmission line are different, matching circuits have to be applied. Even if both impedances are equal, some matching may be required to improve the overall passband performance. Since a CRLH transmission line is equivalent to the central part of a Chebyshev band-pass filter, the impedance matching circuits can be constructed from the corresponding band-pass filter design. This procedure is equivalent to the design of an entire filter and automatically results in good matching performance.

As an example, we have designed a CRLH transmission line of finite length in the balanced case, for example, with 21 series resonators and 20 shunt resonators according to the circuit model in Figure 1(a). The CRLH transmission line is composed of symmetrical unit elements [5], and the simulated voltage reflection coefficients are shown in Figure 4(a). At the center frequency, the impedance match is almost perfect. This is the simplest method; nevertheless large ripples exist near the cut-off frequencies, which considerably affect the passband of the CRLH transmission line. On the other hand, if we design the CRLH transmission line from a 41st-order Chebyshev filter with $0.01 \mathrm{~dB}$ passband 
ripple by the method in Section 3, the impedance match is excellent over the whole passband as shown in Figure 4(b). However, all 41 resonators have to be designed exactly using the required element values.

The trade-off is a hybrid design, in which the periodic structure of the CRLH transmission line is applied, but several resonators near either end are exchanged with those from the corresponding band-pass filter, for example, a 41storder Chebyshev band-pass filter with $0.01 \mathrm{~dB}$ passband ripple. The detailed procedure for our example is as follows: (a) design a 41st-order Chebyshev filter with $0.01 \mathrm{~dB}$ passband ripple and a CRLH transmission line with 41 periodic unit cells with the same cut-off frequencies and characteristic impedance, respectively; (b) choose the first and last three LC resonators from the Chebyshev filter, and place them into the CRLH transmission line at the corresponding positions. The simulated results of the hybrid design with three resonators exchanged at either end are shown in Figure 4(c). A better impedance match compared to Figure 4(a) has been achieved. With the modification of more resonators, better results can be obtained.

If the dual-CRLH transmission line is taken into consideration, a similar procedure can be applied. The passband ripple of a high-order Chebyshev band-stop filter corresponds to the mismatch between the characteristic impedance of a dual-CRLH transmission line and the system impedance.

In the case that even order Chebyshev filters are concerned, the load impedance, which is only dependent on the passband ripple and independent on the filter order, is always different from the source impedance. However, when the passband ripple is small, the load impedance is close to the source impedance. Therefore, there is not much difference between filter element values of even and odd order for low passband ripple and high order of the filter. On the other hand, when the passband ripple is high, the difference between even order and odd filters appears only in a limited number of elements near the load; these can be understood as an impedance matching circuit. Thus, with respect to even order Chebyshev filters, the analysis in this paper is also suitable.

Another point that we would like to stress here is the design flexibility of a CRLH transmission line. Between CRLH transmission lines and Chebyshev band-pass filters, there are three common parameters, that is, two cut-off frequencies and the matching impedance. Besides these parameters, there is one more parameter in the design of band-pass filters, that is, the passband ripple. It reflects the impedance mismatch between CRLH transmission lines and source/load. Once some passband ripple variation is allowed in a limited range, it gives more flexibility in the design of a CRLH transmission line.

\section{Group Delay and Negative Phase Velocity}

Left-handed transmission lines support electromagnetic waves with phase and group velocities that are antiparallel to each other. The apparent backward wave propagation is one

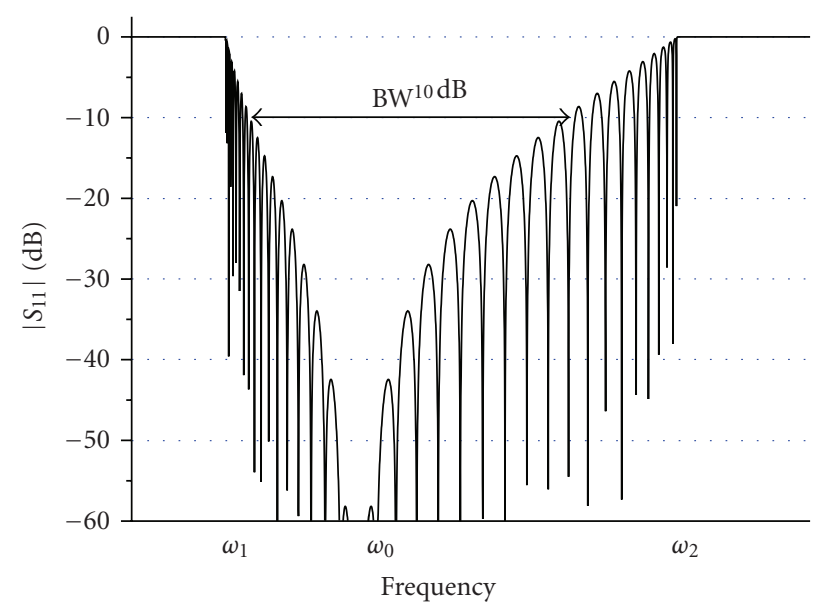

(a)

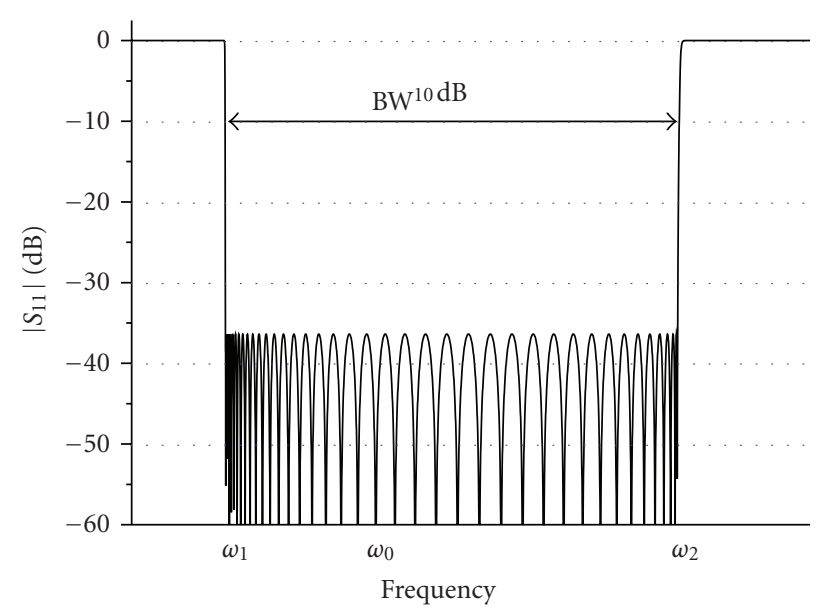

(b)

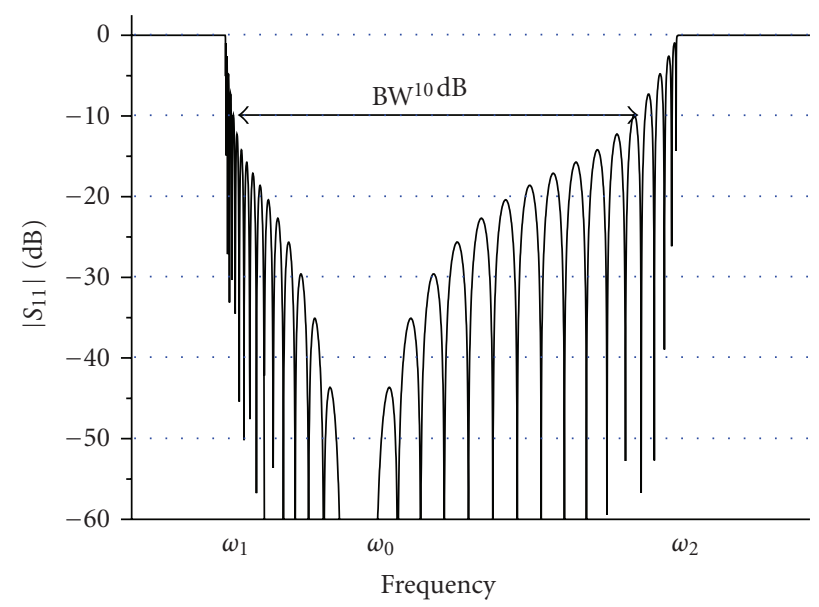

(c)

Figure 4: Comparison of $10 \mathrm{~dB}$ bandwidths between CRLH transmission lines. (a) Conventional CRLH transmission line with 20 symmetric unit elements; (b) CRLH transmission line from 41storder Chebyshev band-pass filter with $0.01 \mathrm{~dB}$ passband ripple; (c) CRLH transmission line with three resonators exchanged at either end. 


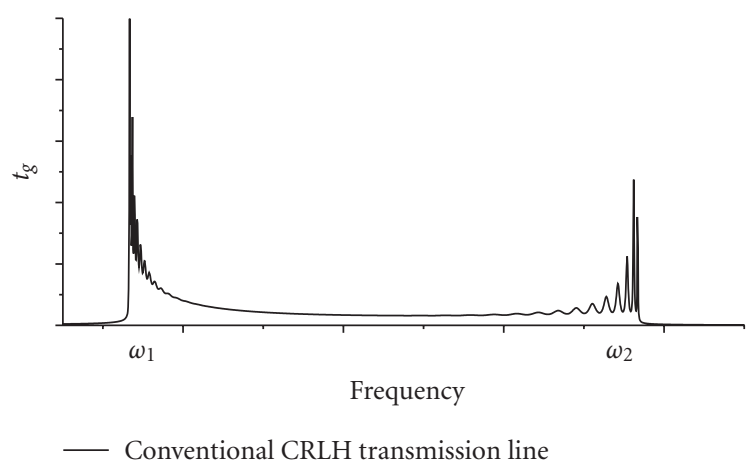

(a)

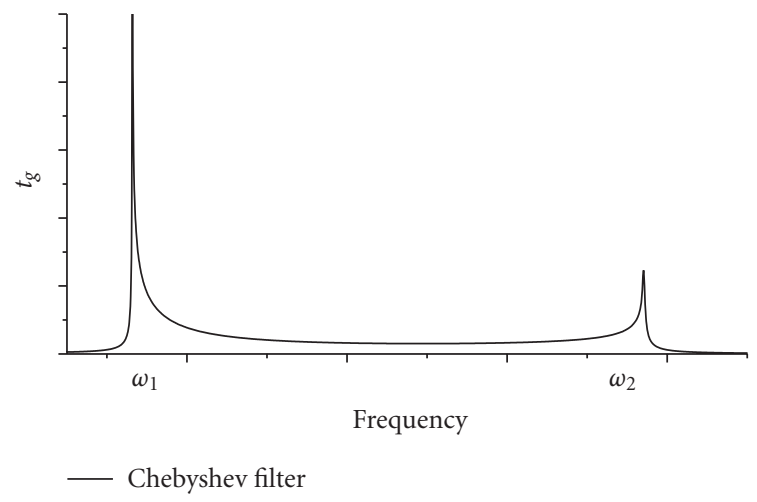

(b)

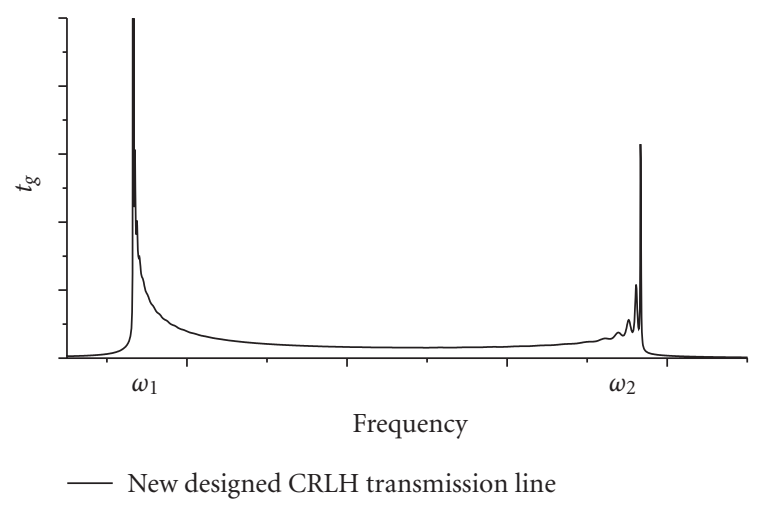

(c)

FIGURE 5: Group delay of a finite length CRLH transmission line with 20 symmetric unit elements, the correspondent 41st-order Chebyshev band-pass filter, and the modified CRLH transmission line with three resonators from the Chebyshev band-pass filter at either end.

key characteristic of CRLH transmission lines. We will show that this does exist with Chebyshev band-pass filters in part of the frequency band due to a similar phase performance compared to CRLH transmission lines.

Group delay, which is an important parameter of a filter, is defined as

$$
t_{g}=-\frac{d \varphi(\omega)}{d \omega}
$$

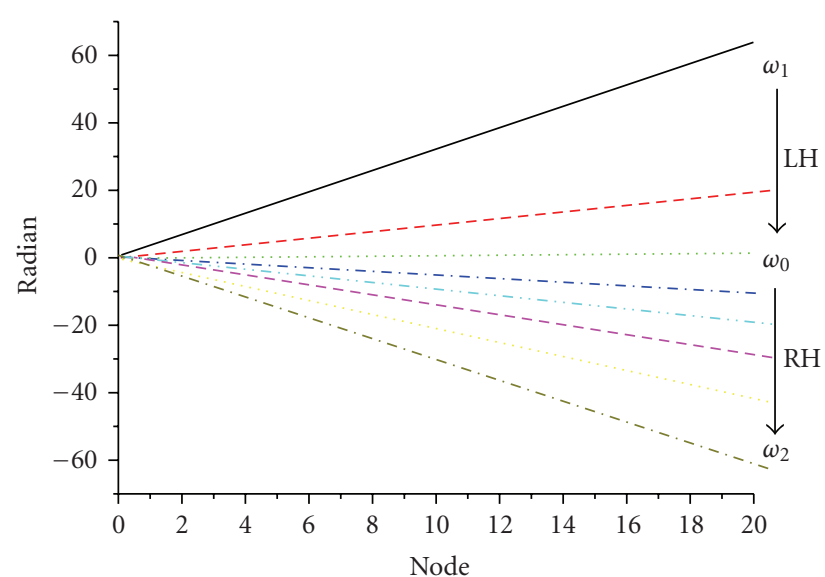

Figure 6: Phase shifts along a 41st-order Chebyshev band-pass filter from the lower cut-off frequency $\omega_{1}$ to the higher cut-off frequency $\omega_{2}$. The left-handed $(\mathrm{LH})$ region is between $\omega_{1}$ and $\omega_{0}$, and the right-handed $(\mathrm{RH})$ region is between $\omega_{0}$ and $\omega_{2}$.

Flat and consistent group delay over frequency is of importance in many microwave systems. A comparison of group delay between a conventional finite length CRLH transmission line, the corresponding Chebyshev band-pass filter, and the modified CRLH transmission line is shown in Figure 5. They all have a similar performance over frequency. There are no ripples in the case of the band-pass filter (Figure 5(b)), while ripples obviously exist around the cut-off frequencies $\omega_{1}$ and $\omega_{2}$ in Figure 5(a). The performance of the new designed CRLH transmission line, as shown in Figure 5(c), lies between the conventional CRLH transmission line and the Chebyshev filter. As can be seen, Chebyshev filters have a better group delay performance compared to conventional, finite length CRLH transmission lines.

When a band-pass filter is built from lumped/ quasilumped elements, the dimensions of each element are much smaller than the wavelength in the passband of the filter. The phase shift along the filter can be calculated based on its equivalent $L C$ circuit. With the example of a 41st-order Chebyshev band-pass filter with $0.01 \mathrm{~dB}$ passband ripple, there are twenty nodes (the connection nodes between series-branch and shunt-branch resonators) in the filter. The phase shifts along those nodes at different frequencies are shown in Figure 6. Similar results are obtained for a conventional CRLH transmission line. At the center frequency $\omega_{0}$, all nodes exhibit the same phase, which is known as the zeroth-order resonance in a conventional CRLH transmission line. In the right-handed region, phase angles decrease along the nodes according to a forward wave propagation. In the left-handed region, phase angles increase along the nodes, approximating a negative phase velocity.

This phase analysis shows the equivalence between a finite length transmission line and a Chebyshev filter also with respect to approaching a negative phase velocity, where the filter has a superior amplitude and group delay performance compared to conventional finite length CRLH transmission lines. 


\section{Conclusions}

The characteristics of finite length CRLH transmission lines and high-order Chebyshev band-pass filters are analyzed, and synthesis formulas based on the matching impedance and cut-off frequencies are shown. From the analysis of element values in Chebyshev prototype lowpass filter, close relations between a CRLH transmission line and a Chebyshev band-pass filter are revealed. It is proven that a CRLH transmission line - in the balanced case - can be considered as the central part of a high-order low passband ripple Chebyshev band-pass filter with identical matching impedance and cutoff frequencies.

The meaning of the passband ripple in a Chebyshev band-pass, which has no obvious counterpart in a CRLH transmission line, corresponds to an impedance mismatch between the CRLH transmission line and the source/load. The formula to compute the passband ripple from the impedance mismatch is presented as well. In addition, impedance matching circuits in finite length CRLH transmission line applications can be design based on the classical filter theory, achieving much better performance.

Phase analysis, including group delay and phase distribution, shows that Chebyshev filters have similar and partly even better phase responses compared to conventional finite length CRLH transmission lines. Negative phase velocity is supported by Chebyshev filters in the same way as with CRHL transmission lines. This confirms the relation between Chebyshev filters and CRLH transmission lines.

Similar results can be obtained for dual-CRLH transmission lines (another kind of negative refractive index transmission lines) and Chebyshev band-stop filters.

Summarizing, this means that a finite length CRLH/dualCRLH transmission line can be designed from a Chebyshev band-pass/band-stop filter. By allowing a reasonable passband ripple, there is even more design freedom. Based on impedance matching, the design from classical filter theory can achieve smooth broadband responses.

First examples for such an approach have already been presented in $[8,9]$ where sections of a CRLH transmission line have been realized as band-pass filters to design antennas with backfire-to-endfire scanning.

Finally, we would like to state that we do not at all want to replace the CRLH transmission line concept by filter theory. The CRLH transmission line concept is very useful to get new insight and to arrive at new design concepts, mainly by phase/dispersion engineering which is generally not at all evident from filter theory, but filter theory may help to improve its performance in the case of finite CRHL transmission line length.

\section{Appendix}

\section{Element Values of a High-Order Prototype Chebyshev Lowpass Filter}

An $N$ th-order prototype Chebyshev lowpass filter with a passband ripple of $L_{A r} \mathrm{~dB}$, a cut-off frequency at $\omega_{0}^{\prime}=1$ and a normalized impedance $g_{0}=1$, has the initial coefficients [14]:

$$
\begin{gathered}
\beta=2 \sinh ^{-1}\left(10^{L_{A r} / 10}-1\right)^{-1 / 2}, \\
\gamma=\sinh \left(\frac{\beta}{2 N}\right)
\end{gathered}
$$

and the corresponding coefficients:

$$
\begin{gathered}
a_{k}=\sin \left[\left(k-\frac{1}{2}\right) \frac{\pi}{N}\right], \\
b_{k}=\gamma^{2}+\sin ^{2}\left(k \frac{\pi}{N}\right) .
\end{gathered}
$$

The element values are $g_{1}=2\left(a_{1} / \gamma\right)$ and $g_{k}=$ $4 a_{k-1} a_{k} / b_{k-1} g_{k-1}$, where $k=2,3, \ldots, N$. Assuming that the filter order is high, that is, $N \gg \max [1, \beta], \gamma$ approaches $\beta / 2 N$.

In the beginning part of the prototype lowpass filter $(k \ll$ $N$ ), the corresponding coefficients are

$$
\begin{gathered}
a_{k} \simeq \frac{(2 k-1) \pi}{2 N}, \\
b_{k} \simeq\left(\frac{\beta}{2 N}\right)^{2}+\left(\frac{k \pi}{N}\right)^{2} .
\end{gathered}
$$

Then the element values of the prototype lowpass filter are

$$
\begin{aligned}
g_{2 k+1}= & j \frac{(4 k+1) \pi}{\beta^{\prime}} \frac{\prod_{m=1}^{k}\left(1-\left(\beta^{\prime 2} /(2 m-1)^{2} \pi^{2}\right)\right)}{\prod_{m=1}^{k}\left(1-\left(\beta^{\prime 2} /(2 m)^{2} \pi^{2}\right)\right)} \\
& \times \prod_{m=1}^{k}\left(\frac{(2 m-1)^{2}}{(2 m)^{2}}\right), \\
g_{2 k+2}= & j \frac{(4 k+3) \beta^{\prime}}{(k-1)^{2} \pi} \frac{\prod_{m=1}^{k}\left(1-\left(\beta^{\prime 2} /(2 m)^{2} \pi^{2}\right)\right)}{\prod_{m=1}^{k+1}\left(1-\left(\beta^{\prime 2} /(2 m-1)^{2} \pi^{2}\right)\right)} \\
& \times \prod_{m=1}^{k}\left(\frac{(2 m)^{2}}{(2 m-1)^{2}}\right),
\end{aligned}
$$

where $\beta^{\prime}=j(\beta / 2)$ and $k=1,2,3, \ldots(k \ll N)$.

The element values can be approximated by the infinite products of the sine and cosine functions and the Wallis' series. Then the element values are

$$
\begin{gathered}
g_{2 k+1} \approx j \frac{2(4 k+1)}{2 k+1} \frac{\cos ^{2}\left(\beta^{\prime} / 2\right)}{\sin \beta^{\prime}} \approx j \frac{4 \cos ^{2}\left(\beta^{\prime} / 2\right)}{\sin \beta^{\prime}}, \\
g_{2 k+2} \approx-j \frac{2 k(4 k+3)}{2(2 k+1)^{2}} \frac{\sin \beta^{\prime}}{\cos ^{2}\left(\beta^{\prime} / 2\right)} \approx-j \frac{\sin \beta^{\prime}}{\cos ^{2}\left(\beta^{\prime} / 2\right)} .
\end{gathered}
$$

Substituting $\beta^{\prime}$ by $j(\beta / 2)$, the element values can be rewritten as

$$
\begin{aligned}
& g_{\text {odd }}=g_{2 k+1} \approx 2 \operatorname{coth} \frac{\beta}{4}=2 \alpha, \\
& g_{\text {even }}=g_{2 k+2} \approx 2 \tanh \frac{\beta}{4}=\frac{2}{\alpha},
\end{aligned}
$$


where $\alpha=\operatorname{coth}(\beta / 4)$. $\beta$ is dependent on the passband ripple $L_{A r} \mathrm{~dB}$ as in (13). When $L_{A r}$ is small, we have $\beta \gg 1$.

Therefore, for a high-order low-ripple $(N \gg \beta \gg 1)$ prototype Chebyshev lowpass filters, we have

$$
g_{\text {odd }} \simeq g_{\text {even }} \simeq 2 .
$$

The element values approach 2 after the beginning parts of the filter.

On the other hand, when the passband ripple is high, that is, $\beta \approx 1$ or $\beta<1$, (A.6) shows that the element value of $g_{i}$ will switch between $g_{\text {odd }}>2$ and $g_{\text {even }}<2$. The adjacent element values satisfy $g_{\text {odd }} \cdot g_{\text {even }} \simeq 4$.

For a high-order Chebyshev prototype lowpass filter, that is, $N \gg \max [1, \beta]$, a general relation between two adjacent element values is obtained from (A.3):

$$
\begin{aligned}
g_{2 k+1} & =\frac{4}{g_{2 k}} \cdot \frac{a_{2 k} a_{2 k+1}}{b_{2 k}} \\
& \approx \frac{4}{g_{2 k}} \cdot \frac{\sin ((4 k+1) \pi / 2 N) \sin ((4 k+3) \pi / 2 N)}{\sin ^{2}(2 k(\pi / N))} .
\end{aligned}
$$

When the element is not close to either end of the filter, that is, $k \gg 1$ and $N-k \gg 1$, we have $g_{2 k} \cdot g_{2 k+1} \simeq 4$.

Thus the sequence of element values keeps $g_{i} \simeq 2$ of a high-order $(N \gg \max [1, \beta])$ Chebyshev prototype lowpass filter with low passband ripple $\left(L_{A r} \ll 1\right)$, when those elements are not close to either end of the filter. Otherwise, when the passband ripple of the filter is high, those element values will keep switching between $g_{\text {odd }}>2$ and $g_{\text {even }}<2$.

For example, we show (Figure 3 ) some calculated results of the element values of Chebyshev lowpass filters. When $N>20,4<k<N-4$, and $L_{A r}=0.01 \mathrm{~dB}$, the maximum relative error of the element values to $g=2$ is less than $8 \%$. If the filter order goes higher and the passband ripple becomes lower, for example, $N>30$ and $L_{A r}=0.001 \mathrm{~dB}$, those errors are less than $6 \%$. Moreover, those errors for elements around the central part of the filter keep always below 3\%.

\section{References}

[1] V. G. Veselago, "The electrodynamics of substances with simultaneously negative values of $\varepsilon$ and $\mu$," Soviet Physics Uspekhi, vol. 10, no. 4, pp. 509-514, 1968.

[2] G. V. Eleftheriades, A. K. Iyer, and P. C. Kremer, "Planar negative refractive index media using periodically L-C loaded transmission lines," IEEE Transactions on Microwave Theory and Techniques, vol. 50, no. 12, pp. 2702-2712, 2002.

[3] A. Grbic and G. V. Eleftheriades, "Experimental verification of backward-wave radiation from a negative refractive index metamaterial," Journal of Applied Physics, vol. 92, no. 10, pp. 5930-5935, 2002.

[4] C. Caloz, H. Okabe, T. Iwai, and T. Itoh, "Transmission line approach of left-handed (LH) materials," in USNC/URSI National Radio Science Meeting, vol. 1, p. 39, San Antonio, Tex, USA, June 2002.

[5] C. Caloz and T. Itoh, Electromagnetic Metamaterials: Transmission Line Theory and Microwave Applications: The Engineering Approach, John Wiley \& Sons, New York, NY, USA, 2006.

[6] A. Lai, C. Caloz, and T. Itoh, "Composite right/left-handed transmission line metamaterials," IEEE Microwave Magazine, vol. 5, no. 3, pp. 34-50, 2004.
[7] C. Caloz, A. Sanada, and T. Itoh, "A novel composite right/left-handed coupled-line directional coupler with arbitrary coupling level and broad bandwidth," IEEE Transactions on Microwave Theory and Techniques, vol. 52, no. 3, pp. 980-992, 2004.

[8] W. Menzel and M. Sathiaseelan, "Frequency scanned antenna array using a suspended stripline negative index transmission line," in Proceedings of the 35th European Microwave Conference (EUMC '05), vol. 1, pp. 253-256, 2005.

[9] W. Menzel and T. Ziqiang, "A fully integrated scanning receiver array," in Proceedings of the 3rd European Radar Conference (EuRAD '06), pp. 61-64, Manchester, UK, September 2007.

[10] G. V. Eleftheriades, "Enabling RF/microwave devices using negative-refractive-index transmission-line metamaterials," Tech. Rep. 312, pp. 57-69, Radio Science URSI Bullet, March 2005.

[11] N. Engheta and R. W. Ziolkowski, "A positive future for double-negative metamaterials," IEEE Transactions on Microwave Theory and Techniques, vol. 53, no. 4, pp. 15351555, 2005.

[12] C. Caloz, "Dual composite right/left-handed (D-CRLH) transmission line metamaterial," IEEE Microwave and Wireless Components Letters, vol. 16, no. 11, pp. 585-587, 2006.

[13] C. Liu and W. Menzel, "On the relation between a negative refractive index transmission line and Chebyshev filters," in Proceedings of the 37th European Microwave Conference (EUMC '07), pp. 704-707, Munich, Germany, October 2007.

[14] G. L. Matthaei, L. Young, and E. M. T. Jones, Microwave Filters, Impedance-Matching Networks, and Coupling Structures, chapter 3, 4, 8, Artech House, Dedham, Mass, USA, 1980. 

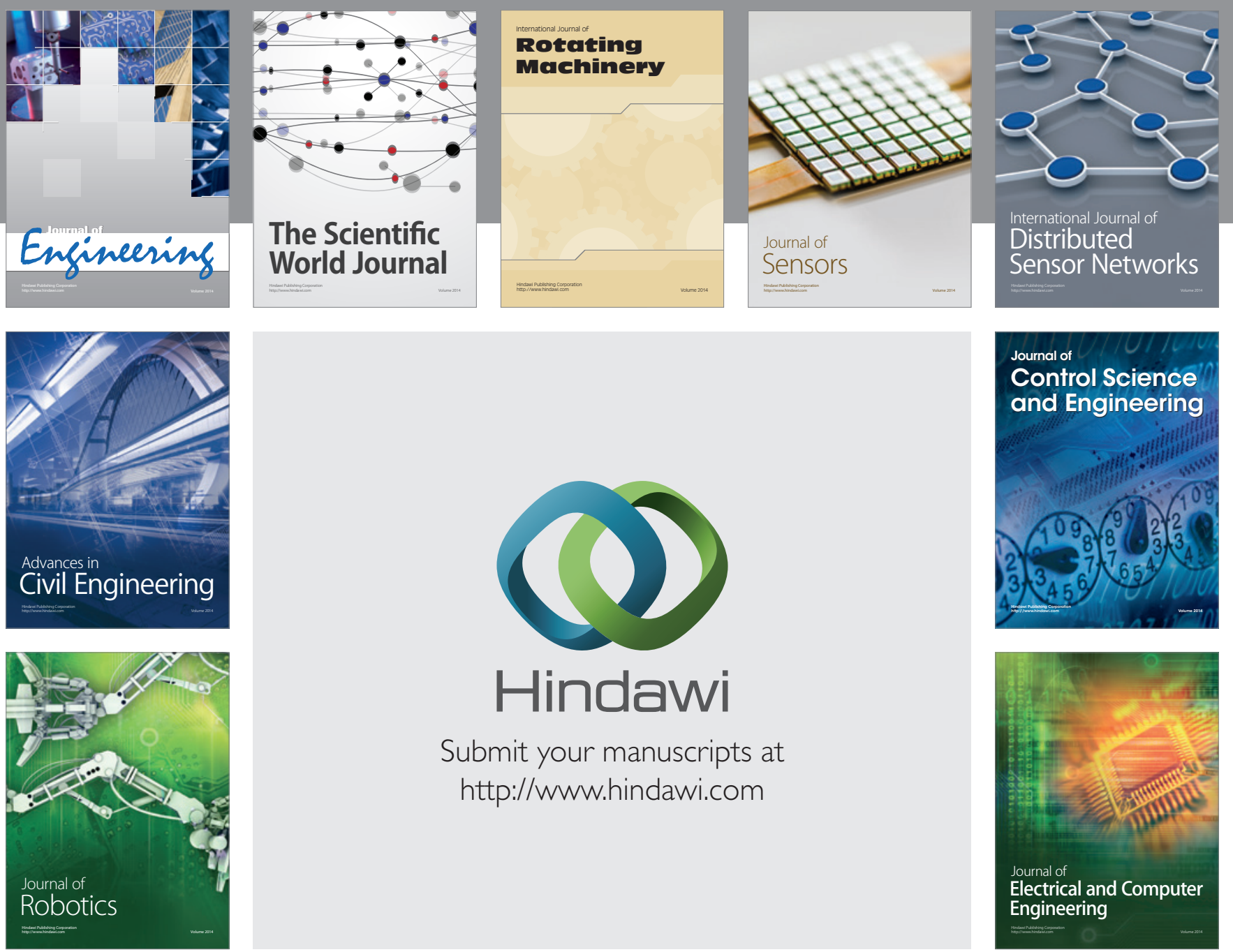

Submit your manuscripts at

http://www.hindawi.com
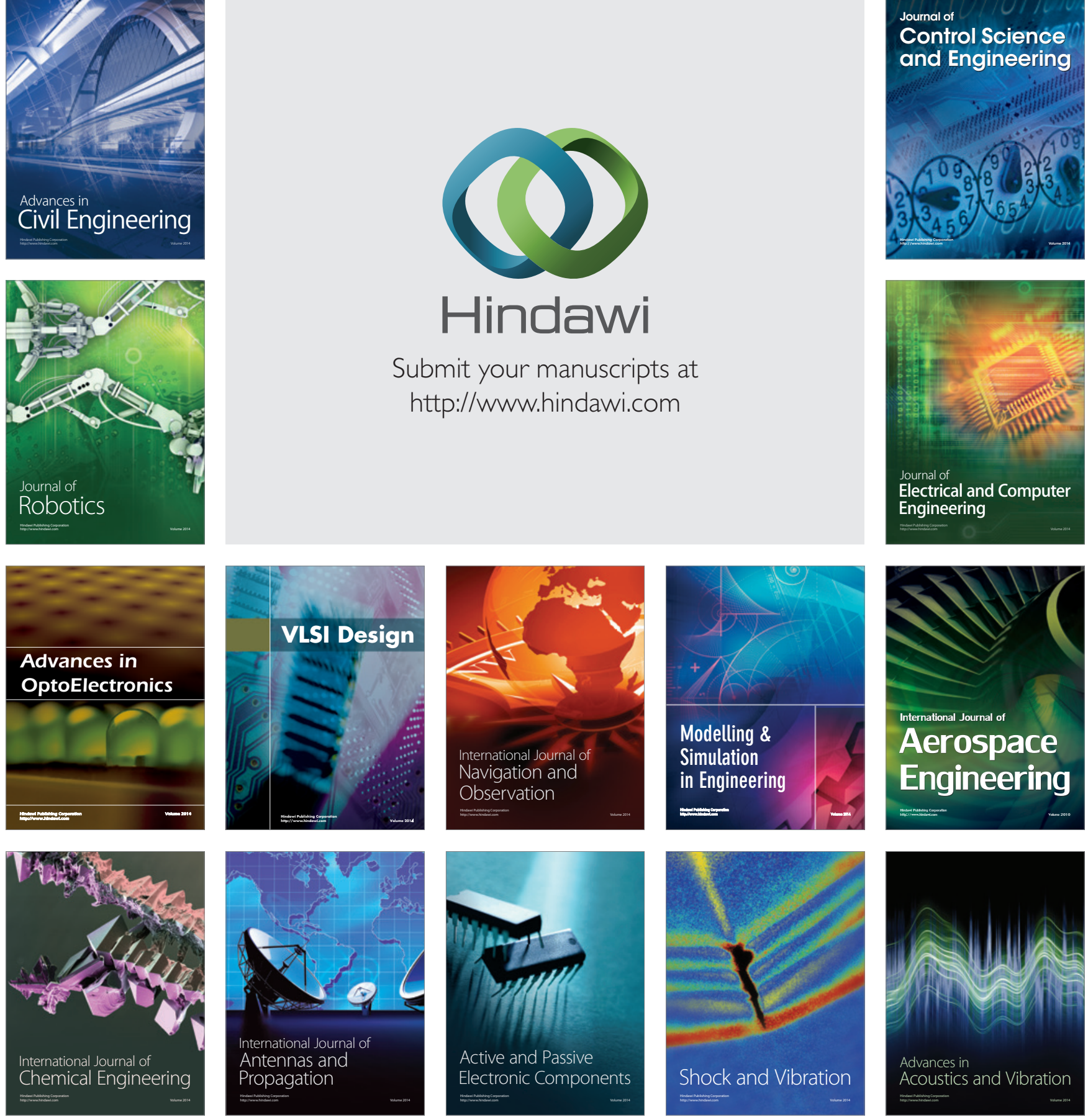